\title{
ON THE PRODUCT AND RATIO OF BESSEL RANDOM VARIABLES
}

\author{
SARALEES NADARAJAH AND ARJUN K. GUPTA
}

Received 20 January 2005

The distributions of products and ratios of random variables are of interest in many areas of the sciences. In this paper, the exact distributions of the product $|X Y|$ and the ratio $|X / Y|$ are derived when $X$ and $Y$ are independent Bessel function random variables. An application of the results is provided by tabulating the associated percentage points.

\section{Introduction}

For given random variables $X$ and $Y$, the distributions of the product $|X Y|$ and the ratio $|X / Y|$ are of interest in many areas of the sciences.

In traditional portfolio selection models, certain cases involve the product of random variables. The best examples of these are in the case of investment in a number of different overseas markets. In portfolio diversification models (see, e.g., Grubel [6]), not only are prices of shares in local markets uncertain, but also the exchange rates are uncertain so that the value of the portfolio in domestic currency is related to a product of random variables. Similarly in models of diversified production by multinationals (see, e.g., Rugman [21]), there are local production uncertainty and exchange rate uncertainty so that profits in home currency are again related to a product of random variables. An entirely different example is drawn from the econometric literature. In making a forecast from an estimated equation, Feldstein [4] pointed out that both the parameter and the value of the exogenous variable in the forecast period could be considered as random variables. Hence, the forecast was proportional to a product of random variables.

An important example of ratios of random variables is the stress-strength model in the context of reliability. It describes the life of a component which has a random strength $Y$ and is subjected to random stress $X$. The component fails at the instant that the stress applied to it exceeds the strength and the component will function satisfactorily whenever $Y>X$. Thus, $\operatorname{Pr}(X<Y)$ is a measure of component reliability. It has many applications especially in engineering concepts such as structures, deterioration of rocket motors, static fatigue of ceramic components, fatigue failure of aircraft structures, and the aging of concrete pressure vessels.

The distributions of $|X Y|$ and $|X / Y|$ have been studied by several authors especially when $X$ and $Y$ are independent random variables and come from the same family. With 
respect to products of random variables, see Sakamoto [22] for uniform family, Harter [7] and Wallgren [28] for Student's $t$ family, Springer and Thompson [24] for normal family, Stuart [26] and Podolski [14] for gamma family, Steece [25], Bhargava and Khatri [3], and Tang and Gupta [27] for beta family, Abu-Salih [1] for power function family, and Malik and Trudel [11] for exponential family (see also Rathie and Rohrer [20] for a comprehensive review of known results). With respect to ratios of random variables, see Marsaglia [12] and Korhonen and Narula [9] for normal family, Press [15] for Student's $t$ family, Basu and Lochner [2] for Weibull family, Shcolnick [23] for stable family, Hawkins and Han [8] for noncentral chi-square family, Provost [16] for gamma family, and PhamGia [13] for beta family.

In this paper, we study the exact distributions of $|X Y|$ and $|X / Y|$ when $X$ and $Y$ are independent Bessel function random variables with pdfs

$$
\begin{aligned}
& f_{X}(x)=\frac{|x|^{m}}{\sqrt{\pi} 2^{m} b^{m+1} \Gamma(m+1 / 2)} K_{m}\left(\left|\frac{x}{b}\right|\right), \\
& f_{Y}(y)=\frac{|y|^{n}}{\sqrt{\pi} 2^{n} \beta^{n+1} \Gamma(n+1 / 2)} K_{n}\left(\left|\frac{y}{\beta}\right|\right),
\end{aligned}
$$

respectively, for $-\infty<x<\infty,-\infty<y<\infty, b>0, \beta>0, m>1$, and $n>1$, where

$$
K_{\nu}(x)=\frac{\sqrt{\pi} x^{\nu}}{2^{\nu} \Gamma(\nu+1 / 2)} \int_{1}^{\infty}\left(t^{2}-1\right)^{\nu-1 / 2} \exp (-x t) d t
$$

is the modified Bessel function of the third kind. Tabulations of the associated percentage points are also provided.

Bessel function distributions have found applications in a variety of areas that range from image and speech recognition and ocean engineering to finance. They are rapidly becoming distributions of first choice whenever "something" heavier than Gaussian tails is observed in the data. Some examples are as follows (see Kotz et al. [10] for further applications).

(1) In communication theory, $X$ and $Y$ could represent the random noises corresponding to two different signals.

(2) In ocean engineering, $X$ and $Y$ could represent distributions of navigation errors.

(3) In finance, $X$ and $Y$ could represent distributions of log-returns of two different commodities.

(4) In image and speech recognition, $X$ and $Y$ could represent "input" distributions. In each of the examples above, it will be of interest to study the distribution of the ratio $|X / Y|$. For example, in communication theory, $|X / Y|$ could represent the relative strength of the two different signals. In ocean engineering, $|X / Y|$ could represent the relative safety of navigation. In finance, $|X / Y|$ could represent the relative popularity of the two different commodities. The distribution of the product $|X Y|$ is considered here for completeness. 
The exact expressions for the distributions of the product and ratio are given in Sections 2 and 3 of the paper. The calculations involve the generalized hypergeometric function defined by

$$
{ }_{p} F_{q}\left(a_{1}, \ldots, a_{p} ; b_{1}, \ldots, b_{q} ; x\right)=\sum_{k=0}^{\infty} \frac{\left(a_{1}\right)_{k}\left(a_{2}\right)_{k} \cdots\left(a_{p}\right)_{k}}{\left(b_{1}\right)_{k}\left(b_{2}\right)_{k} \cdots\left(b_{q}\right)_{k}} \frac{x^{k}}{k !}
$$

where $(e)_{k}=e(e+1) \cdots(e+k-1)$ denotes the ascending factorial. We also need the following important lemmas.

Lemma 1.1 (Prudnikov et al. [18, equation (2.16.33.5), Volume 2]). For $b>0$ and $c>0$,

$$
\begin{aligned}
& \int_{0}^{\infty} x^{\alpha-1} K_{\mu}\left(\frac{b}{x}\right) K_{\nu}(c x) d x \\
& =2^{\alpha-2 \mu-3} b^{\mu} c^{\mu-\alpha} \Gamma(-\mu) \Gamma\left(\frac{\alpha+\nu-\mu}{2}\right) \Gamma\left(\frac{\alpha-\nu-\mu}{2}\right) \\
& \times{ }_{0} F_{3}\left(1+\mu, 1+\frac{\mu-\nu-\alpha}{2}, 1+\frac{\nu+\mu-\alpha}{2} ; \frac{b^{2} c^{2}}{16}\right) \\
& +2^{\alpha+2 \mu-3} b^{-\mu} c^{-\mu-\alpha} \Gamma(\mu) \Gamma\left(\frac{\alpha+\nu+\mu}{2}\right) \Gamma\left(\frac{\alpha-\nu+\mu}{2}\right) \\
& \times_{0} F_{3}\left(1-\mu, 1-\frac{\alpha+\mu+v}{2}, 1-\frac{\alpha+\mu-\nu}{2} ; \frac{b^{2} c^{2}}{16}\right) \\
& +2^{-\alpha-2 \nu-3} b^{\alpha+\nu} c^{\nu} \Gamma(-\nu) \Gamma\left(\frac{\mu-\nu-\alpha}{2}\right) \Gamma\left(-\frac{\mu+\nu+\alpha}{2}\right) \\
& \times_{0} F_{3}\left(1+\nu, 1+\frac{\alpha+\nu-\mu}{2}, 1+\frac{\alpha+\mu+\nu}{2} ; \frac{b^{2} c^{2}}{16}\right) \\
& +2^{2 \nu-\alpha-3} b^{\alpha-v} c^{-\nu} \Gamma(\nu) \Gamma\left(\frac{\mu+\nu-\alpha}{2}\right) \Gamma\left(\frac{\nu-\mu-\alpha}{2}\right) \\
& \times_{0} F_{3}\left(1-v, 1+\frac{\alpha-\mu-v}{2}, 1+\frac{\alpha+\mu-v}{2} ; \frac{b^{2} c^{2}}{16}\right) \text {. }
\end{aligned}
$$

Lemma 1.2 (Gradshteyn and Ryzhik [5, equation (6.576.4)]). For $a+b>0$ and $\lambda<1-$ $\mu-v$,

$$
\begin{aligned}
\int_{0}^{\infty} x^{-\lambda} K_{\mu}(a x) K_{\nu}(b x) d x= & \frac{2^{-2-\lambda} a^{-\nu+\lambda-1} b^{\nu}}{\Gamma(1-\lambda)} \Gamma\left(\frac{1-\lambda+\mu+\nu}{2}\right) \Gamma\left(\frac{1-\lambda-\mu+\nu}{2}\right) \\
& \times \Gamma\left(\frac{1-\lambda+\mu-\nu}{2}\right) \Gamma\left(\frac{1-\lambda-\mu-\nu}{2}\right) \\
& \times{ }_{2} F_{1}\left(\frac{1-\lambda+\mu+\nu}{2}, \frac{1-\lambda-\mu+\nu}{2} ; 1-\lambda ; 1-\frac{b^{2}}{a^{2}}\right) .
\end{aligned}
$$


2980 On the product and ratio of Bessel random variables

Lemma 1.3 (Prudnikov et al. [19, equation (2.21.1.14), Volume 3]). For $y>0, \beta>0$, $\alpha+\beta<1+a$, and $\alpha+\beta<1+b$,

$$
\begin{aligned}
& \int_{y}^{\infty} x^{\alpha-1}(x-y)^{\beta-1}{ }_{2} F_{1}(a, b ; c ; 1-w x) d x \\
&= w^{-a} y^{\alpha+\beta-a-1} \frac{\Gamma(c) \Gamma(b-a) \Gamma(\beta) \Gamma(a-\alpha-\beta+1)}{\Gamma(b) \Gamma(c-a) \Gamma(a-\alpha+1)} \\
& \times{ }_{3} F_{2}\left(a, c-b, a-\alpha-\beta+1 ; a-\alpha+1, a-b+1 ; \frac{1}{w y}\right) \\
&+w^{-b} y^{\alpha+\beta-b-1} \frac{\Gamma(c) \Gamma(a-b) \Gamma(\beta) \Gamma(b-\alpha-\beta+1)}{\Gamma(a) \Gamma(c-b) \Gamma(b-\alpha+1)} \\
& \times{ }_{3} F_{2}\left(b, c-a, b-\alpha-\beta+1 ; b-a+1, b-\alpha+1 ; \frac{1}{w y}\right) .
\end{aligned}
$$

Lemma 1.4 (Prudnikov et al. [19, equation (2.22.2.1), Volume 3]). For $a>0, \alpha>0$, $\beta>0$, and $p \leq q+1$,

$$
\begin{aligned}
& \int_{0}^{a} x^{\alpha-1}(a-x)^{\beta-1}{ }_{p} F_{q}\left(a_{1}, \ldots, a_{p} ; b_{1}, \ldots, b_{q} ; w x\right) d x \\
& \quad=a^{\alpha+\beta-1} B(\alpha, \beta)_{p+1} F_{q+1}\left(a_{1}, \ldots, a_{p}, \alpha ; b_{1}, \ldots, b_{q}, \alpha+\beta ; a w\right) .
\end{aligned}
$$

Further properties of the generalized hypergeometric function can be found in Prudnikov et al. [17, 18, 19] and Gradshteyn and Ryzhik [5].

\section{Product}

Theorem 2.1 derives explicit expressions for the distribution of $|X Y|$ in terms of the ${ }_{0} F_{3}$ and ${ }_{1} F_{4}$ hypergeometric functions.

Theorem 2.1. Suppose that $X$ and $Y$ are distributed according to (1.1) and (1.2), respectively. The pdf and the cdf of $Z=|X Y|$ can be expressed as

$$
\begin{gathered}
f_{Z}(z)=K\left\{2^{n-3 m-1} b^{-m} \beta^{n-2 m} \Gamma^{2}(-m) \Gamma(n-m) z^{2 m} C_{1}(z)-2^{m+n} b^{m} \beta^{n} C \Gamma(m) \Gamma(n) C_{2}(z)\right. \\
\left.+2^{m-3 n-1} b^{m-2 n} \beta^{-n} \Gamma^{2}(-n) \Gamma(m-n) z^{2 n} C_{3}(z)\right\}
\end{gathered}
$$

$$
\begin{gathered}
F_{Z}(z)=K\left\{2^{n-3 m-1} b^{-m} \beta^{n-2 m} \Gamma^{2}(-m) \Gamma(n-m) \frac{z^{2 m+1}}{2 m+1} C_{4}(z)-2^{m+n} b^{m} \beta^{n} C \Gamma(m) \Gamma(n) z C_{5}(z)\right. \\
\left.+2^{m-3 n-1} b^{m-2 n} \beta^{-n} \Gamma^{2}(-n) \Gamma(m-n) \frac{z^{2 n+1}}{2 n+1} C_{6}(z)\right\},
\end{gathered}
$$


where

$$
\begin{aligned}
C_{1}(z) & ={ }_{0} F_{3}\left(1+m, 1+m-n, 1+m ; \frac{z^{2}}{16 b^{2} \beta^{2}}\right), \\
C_{2}(z) & ={ }_{0} F_{3}\left(1-m, 1-n, 1 ; \frac{z^{2}}{16 b^{2} \beta^{2}}\right), \\
C_{3}(z) & ={ }_{0} F_{3}\left(1+n, 1+n-m, 1+n ; \frac{z^{2}}{16 b^{2} \beta^{2}}\right), \\
C_{4}(z) & ={ }_{1} F_{4}\left(\frac{1}{2}+m ; 1+m, 1+m-n, 1+m, \frac{3}{2}+m ; \frac{z^{2}}{16 b^{2} \beta^{2}}\right), \\
C_{5}(z) & ={ }_{1} F_{4}\left(\frac{1}{2} ; 1-m, 1-n, 1, \frac{3}{2} ; \frac{z^{2}}{16 b^{2} \beta^{2}}\right), \\
C_{6}(z) & ={ }_{1} F_{4}\left(\frac{1}{2}+n ; 1+n, 1+n-m, 1+n, \frac{3}{2}+n ; \frac{z^{2}}{16 b^{2} \beta^{2}}\right), \\
\frac{1}{K} & =\pi 2^{m+n} b^{m+1} \beta^{n+1} \Gamma\left(m+\frac{1}{2}\right) \Gamma\left(n+\frac{1}{2}\right),
\end{aligned}
$$

and $C$ denotes Euler's constant.

Proof. The pdf of $|X Y|$ can be expressed as

$$
\begin{aligned}
f_{Z}(z) & =4 \int_{0}^{\infty} \frac{1}{y} f_{X}\left(\frac{z}{y}\right) f_{Y}(y) d y \\
& =4 \int_{0}^{\infty} \frac{1}{y} \frac{|z / y|^{m}}{\sqrt{\pi} 2^{m} b^{m+1} \Gamma(m+1 / 2)} K_{m}\left(\left|\frac{z}{b y}\right|\right) \frac{|y|^{n}}{\sqrt{\pi} 2^{n} \beta^{n+1} \Gamma(n+1 / 2)} K_{n}\left(\left|\frac{y}{\beta}\right|\right) d y \\
& =\frac{z^{m} I(m, n)}{\pi 2^{m+n-2} b^{m+1} \beta^{n+1} \Gamma(m+1 / 2) \Gamma(n+1 / 2)},
\end{aligned}
$$

where $I(m, n)$ denotes the integral

$$
I(m, n)=\int_{0}^{\infty} y^{n-m-1} K_{m}\left(\frac{z}{b y}\right) K_{n}\left(\frac{y}{\beta}\right) d y .
$$

The result in (2.1) follows by direct application of Lemma 1.1 to calculate $I(m, n)$. The cdf of $Z$ can be expressed as

$$
\begin{aligned}
F_{Z}(z)=K & \left\{2^{n-3 m-1} b^{-m} \beta^{n-2 m} \Gamma^{2}(-m) \Gamma(n-m) \int_{0}^{z} w^{2 m} C_{1}(w) d w\right. \\
& -2^{m+n} b^{m} \beta^{n} C \Gamma(m) \Gamma(n) \int_{0}^{z} C_{2}(w) d w \\
& \left.+2^{m-3 n-1} b^{m-2 n} \beta^{-n} \Gamma^{2}(-n) \Gamma(m-n) \int_{0}^{z} w^{2 n} C_{3}(w) d w\right\} .
\end{aligned}
$$

The result in (2.2) follows by applying Lemma 1.4 to calculate the three integrals in (2.6). 
2982 On the product and ratio of Bessel random variables

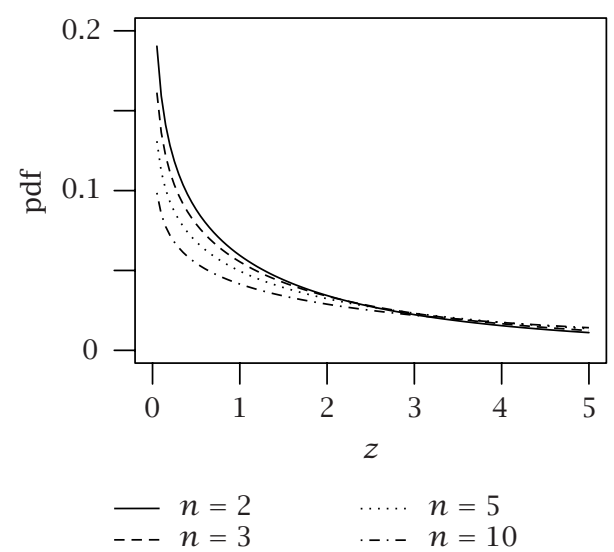

(a)

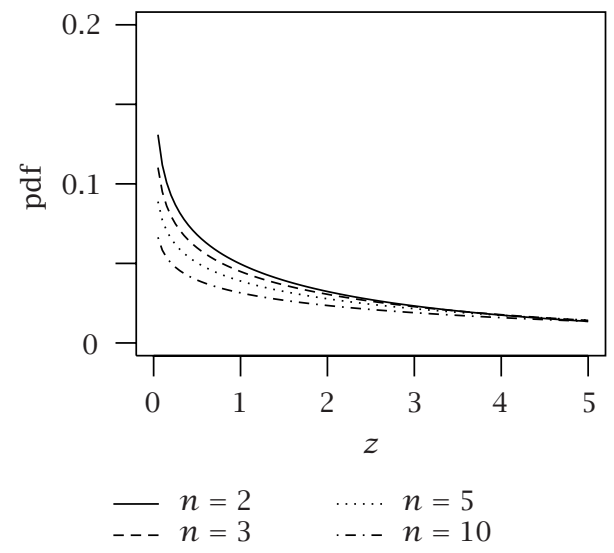

(c)

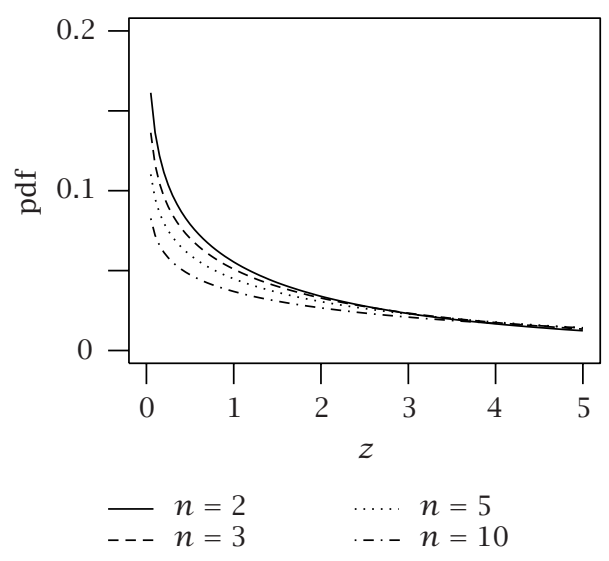

(b)

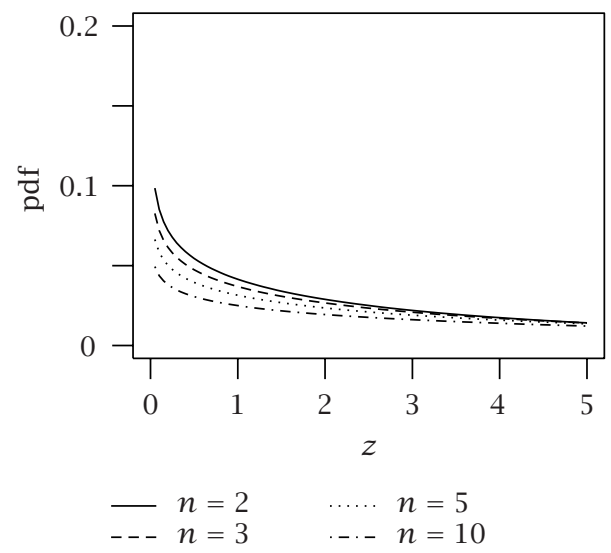

(d)

Figure 2.1. Plots of the pdf (2.1) for $b=1, \beta=1$, and (a) $m=2$; (b) $m=3$; (c) $m=5$; and (d) $m=10$.

Figure 2.1 illustrates possible shapes of the pdf (2.1) for selected values of $m$ and $n$. The four curves in each plot correspond to selected values of $n$. The effect of the parameters is evident.

\section{Ratio}

Theorem 3.1 derives explicit expressions for the distribution of $|X / Y|$ in terms of the ${ }_{2} F_{1}$ and ${ }_{3} F_{2}$ hypergeometric functions. 
Theorem 3.1. Suppose that $X$ and $Y$ are distributed according to (1.1) and (1.2), respectively. The $p d f$ and the cdf of $Z=|X / Y|$ can be expressed as

$$
\begin{aligned}
& f_{Z}(z)=\frac{2 L(\beta / b)^{-2 n-1}}{m+n+1} z^{-2 n-2} D_{1}(z), \\
& F_{Z}(z)=2 L \Gamma(m+n+1)\left\{\frac{\Gamma(-m) D_{2}(z)}{(2 m+1) \Gamma(n+1)}+\frac{\beta z D_{3}(z)}{m b \Gamma(m+n+1)}\right\},
\end{aligned}
$$

where

$$
\begin{gathered}
D_{1}(z)={ }_{2} F_{1}\left(m+n+1, n+1 ; m+n+2 ; 1-\frac{b^{2}}{\beta^{2} z^{2}}\right), \\
D_{2}(z)={ }_{2} F_{1}\left(m+n+1, m+\frac{1}{2} ; m+\frac{3}{2} ; \frac{\beta^{2} z^{2}}{b^{2}}\right), \\
D_{3}(z)={ }_{3} F_{2}\left(n+1,1, \frac{1}{2} ; 1-m, \frac{3}{2} ; \frac{\beta^{2} z^{2}}{b^{2}}\right), \\
L=\frac{\Gamma(m+1) \Gamma(n+1)}{\pi \Gamma(m+1 / 2) \Gamma(n+1 / 2)} .
\end{gathered}
$$

Proof. The pdf of $Z=|X / Y|$ can be expressed as

$$
\begin{aligned}
f_{Z}(z) & =4 \int_{0}^{\infty} y f_{X}(y z) f_{Y}(y) d y \\
& =4 \int_{0}^{\infty} y \frac{(y z)^{m}}{\sqrt{\pi} 2^{m} \Gamma(m+1 / 2)} K_{m}(y z) \frac{y^{n}}{\sqrt{\pi} 2^{n} \Gamma(n+1 / 2)} K_{n}(y) d y \\
& =\frac{z^{m} I(m, n)}{\pi 2^{m+n-2} \Gamma(m+1 / 2) \Gamma(n+1 / 2)},
\end{aligned}
$$

where $I(m, n)$ denotes the integral

$$
I(m, n)=\int_{0}^{\infty} y^{m+n+1} K_{m}(y z) K_{n}(y) d y
$$

The result in (3.1) follows by direct application of Lemma 1.2 to calculate $I(m, n)$. The cdf of $Z$ can be expressed as

$$
\begin{aligned}
F_{Z}(z) & =\frac{2 L(\beta / b)^{-2 n-1}}{m+n+1} \int_{0}^{z} w^{-2 n-2} D_{1}(w) d w \\
& =\frac{L}{m+n+1} \int_{b^{2} /(\beta z)^{2}}^{\infty} x_{2}^{n-1 / 2} F_{1}(m+n+1, n+1 ; m+n+2 ; 1-x) d x,
\end{aligned}
$$

which follows by setting $x=(\beta w / b)^{-2}$. The result in (3.2) follows by applying Lemma 1.3 to calculate the integral in (3.6). 
2984 On the product and ratio of Bessel random variables

Using special properties of the ${ }_{2} F_{1}$ hypergeometric function, one can derive other equivalent forms and elementary forms for the pdf of $Z=|X / Y|$. This is illustrated in the corollaries below.

Corollary 3.2. The pdf given by (3.1) can be expressed in the equivalent forms

$$
\begin{aligned}
& f_{Z}(z)=\frac{2 \beta(\beta z / b)^{2 m} \Gamma(m+1) \Gamma(n+1)}{b \pi(m+n+1) \Gamma(m+1 / 2) \Gamma(n+1 / 2)}{ }_{2} F_{1}\left(m+n+1, m+1 ; m+n+2 ; 1-\frac{\beta^{2} z^{2}}{b^{2}}\right), \\
& f_{Z}(z)=\frac{2 \beta \Gamma(m+1) \Gamma(n+1)}{b \pi(m+n+1) \Gamma(m+1 / 2) \Gamma(n+1 / 2)}{ }_{2} F_{1}\left(n+1,1 ; m+n+2 ; 1-\frac{\beta^{2} z^{2}}{b^{2}}\right), \\
& f_{Z}(z)=\frac{2 \beta(\beta z / b)^{-2} \Gamma(m+1) \Gamma(n+1)}{b \pi(m+n+1) \Gamma(m+1 / 2) \Gamma(n+1 / 2)}{ }_{2} F_{1}\left(1, m+1 ; m+n+2 ; 1-\frac{b^{2}}{\beta^{2} z^{2}}\right) .
\end{aligned}
$$

COROLlary 3.3. If $m \geq 2$ and $n \geq 2$ are integers, then (3.1) can be reduced to the elementary form

$$
\begin{aligned}
f_{Z}(z)= & \frac{2 \beta \Gamma(m+1) \Gamma(m+n+1)}{b \pi\left(\beta^{2} z^{2} / b^{2}-1\right) \Gamma(m+1 / 2) \Gamma(n+1 / 2)} \\
& \times\left[\sum_{k=1}^{n} \frac{(n-k) !\left(1-\beta^{2} z^{2} / b^{2}\right)^{1-k}}{(m+n+1-k) !}+\frac{\left(1-\beta^{2} z^{2} / b^{2}\right)^{1-n}}{m ! \beta^{2} z^{2} / b^{2}}\right. \\
& \left.\times\left\{-2\left(1-\frac{b^{2}}{\beta^{2} z^{2}}\right)^{-m-1} \log \left(\frac{\beta z}{b}\right)+\sum_{k=1}^{m} \frac{\left(1-b^{2} /\left(\beta^{2} z^{2}\right)\right)^{-k}}{m+1-k}\right\}\right] .
\end{aligned}
$$

Corollary 3.4. If $m-1 / 2 \geq 1$ and $n-1 / 2 \geq 1$ are integers, then (3.1) can be reduced to the elementary form

$$
\begin{aligned}
f_{Z}(z)= & \frac{2 b\left(1-\beta^{2} z^{2} / b^{2}\right)^{-m-n-1} \Gamma(m+1) \Gamma(n+1)}{\beta z^{2} \pi(m+n+1)(-m-1)_{m+n+2} \Gamma(m+1 / 2) \Gamma(n+1 / 2)} \\
& \times\left[\Gamma(-m)\left(\frac{\beta z}{b}\right)^{2 m+2}+\sum_{k=1}^{m+n+1}(-m-1)_{k}(-1)^{k}\left(1-\frac{b^{2}}{\beta^{2} z^{2}}\right)^{k-1}(\beta z / b)^{2 k}\right] .
\end{aligned}
$$

\section{Percentiles}

Figure 4.1 illustrates possible shapes of the pdf (3.1) for selected values of $m$ and $n$. The four curves in each plot correspond to selected values of $n$. The effect of the parameters is evident.

In this section, we provide tabulations of percentage points associated with the derived distributions of $|X Y|$ and $|X / Y|$. These values are obtained by numerically solving 


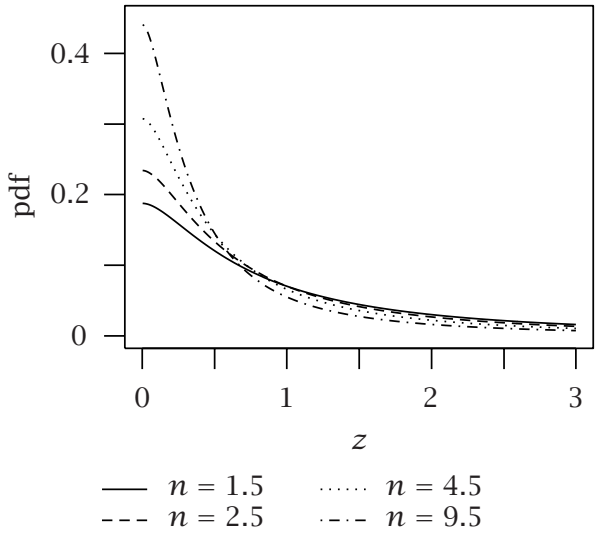

(a)

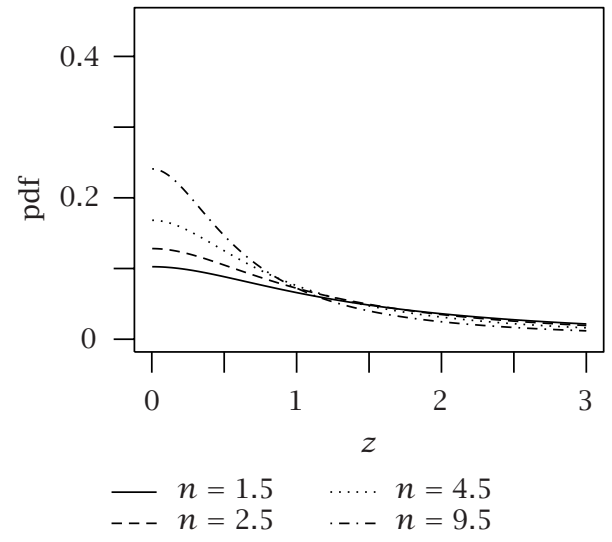

(c)

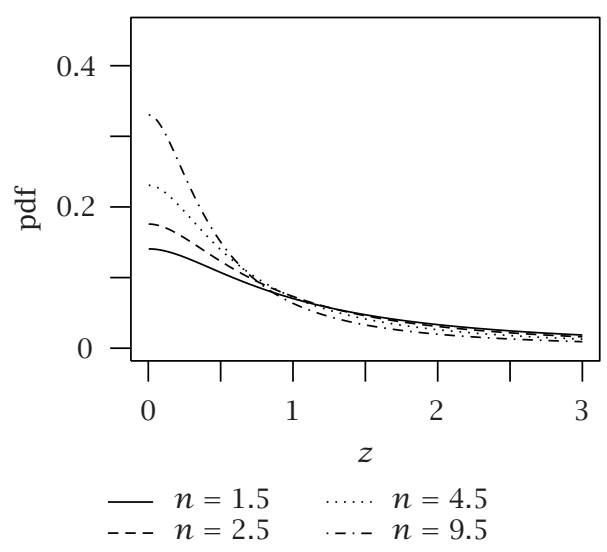

(b)

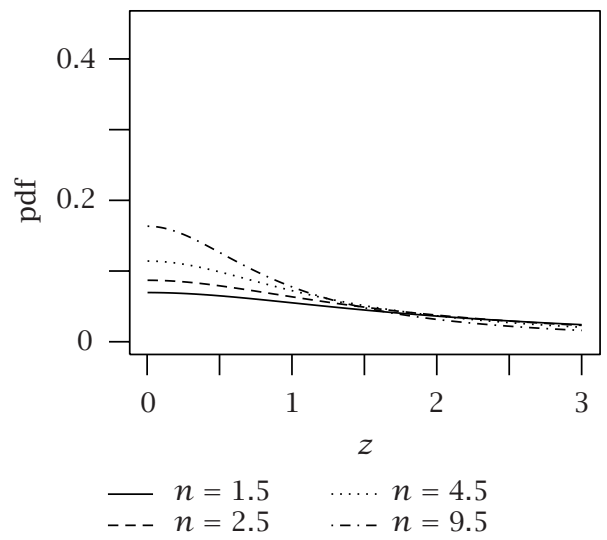

(d)

Figure 4.1. Plots of the pdf (3.1) for $b=1, \beta=1$, and (a) $m=1.5$; (b) $m=2.5$; (c) $m=4.5$; and (d) $m=9.5$.

the equations

$$
\begin{gathered}
K\left\{2^{n-3 m-1} b^{-m} \beta^{n-2 m} \Gamma^{2}(-m) \Gamma(n-m) \frac{z_{p}^{2 m+1}}{2 m+1} C_{4}\left(z_{p}\right)-2^{m+n} b^{m} \beta^{n} C \Gamma(m) \Gamma(n) z_{p} C_{5}\left(z_{p}\right)\right. \\
\left.+2^{m-3 n-1} b^{m-2 n} \beta^{-n} \Gamma^{2}(-n) \Gamma(m-n) \frac{z_{p}^{2 n+1}}{2 n+1} C_{6}\left(z_{p}\right)\right\}=p \\
2 L \Gamma(m+n+1)\left\{\frac{\Gamma(-m) D_{2}\left(z_{p}\right)}{(2 m+1) \Gamma(n+1)}+\frac{\beta z_{p} D_{3}\left(z_{p}\right)}{m b \Gamma(m+n+1)}\right\}=p .
\end{gathered}
$$


2986 On the product and ratio of Bessel random variables

Table 4.1. Percentage points $z_{p}$ of $Z=|X Y|$.

\begin{tabular}{|c|c|c|c|c|c|c|c|}
\hline$m$ & $n$ & $p=0.6$ & $p=0.7$ & $p=0.8$ & $p=0.9$ & $p=0.95$ & $p=0.99$ \\
\hline 2 & 2 & 0.02903867 & 0.0609406 & 0.1328627 & 0.3470357 & 0.6927036 & 2.098452 \\
\hline 2 & 3 & 0.07362923 & 0.1376486 & 0.2682605 & 0.6146536 & 1.130666 & 3.038813 \\
\hline 2 & 4 & 0.1100132 & 0.1998708 & 0.3777723 & 0.827011 & 1.465626 & 3.755526 \\
\hline 2 & 5 & 0.1408100 & 0.2514674 & 0.4678341 & 1.000369 & 1.752475 & 4.380899 \\
\hline 2 & 6 & 0.1673438 & 0.2968515 & 0.5449642 & 1.151051 & 1.991074 & 4.848271 \\
\hline 2 & 7 & 0.1906657 & 0.3359331 & 0.6139745 & 1.284624 & 2.218128 & 5.323573 \\
\hline 2 & 8 & 0.2125461 & 0.372798 & 0.6770319 & 1.415690 & 2.416419 & 5.745124 \\
\hline 2 & 9 & 0.2300810 & 0.4031021 & 0.7305227 & 1.517905 & 2.591786 & 6.145533 \\
\hline 3 & 3 & 0.1744381 & 0.2938927 & 0.5183827 & 1.057357 & 1.798354 & 4.335123 \\
\hline 3 & 4 & 0.2572388 & 0.4184127 & 0.711219 & 1.388183 & 2.282625 & 5.225012 \\
\hline 3 & 5 & 0.3263338 & 0.5227913 & 0.870987 & 1.660568 & 2.691937 & 6.044048 \\
\hline 3 & 6 & 0.3860577 & 0.6124857 & 1.011584 & 1.908407 & 3.049341 & 6.677126 \\
\hline 3 & 7 & 0.4394194 & 0.6929521 & 1.137077 & 2.125184 & 3.382392 & 7.385786 \\
\hline 3 & 8 & 0.4865918 & 0.7648362 & 1.249936 & 2.318062 & 3.661295 & 7.867155 \\
\hline 3 & 9 & 0.5306344 & 0.8335735 & 1.356932 & 2.498872 & 3.923604 & 8.372842 \\
\hline 4 & 4 & 0.3773593 & 0.593275 & 0.972541 & 1.816783 & 2.900670 & 6.372728 \\
\hline 4 & 5 & 0.4760256 & 0.7359029 & 1.184584 & 2.162823 & 3.404096 & 7.3069 \\
\hline 4 & 6 & 0.5626443 & 0.8623236 & 1.371957 & 2.474747 & 3.845828 & 8.08524 \\
\hline 4 & 7 & 0.638364 & 0.9744115 & 1.541777 & 2.752022 & 4.246468 & 8.858037 \\
\hline 4 & 8 & 0.7085111 & 1.076598 & 1.696747 & 3.004645 & 4.632417 & 9.46566 \\
\hline 4 & 9 & 0.7702856 & 1.166607 & 1.830414 & 3.240364 & 4.958135 & 10.19733 \\
\hline 5 & 5 & 0.6033154 & 0.9178398 & 1.450584 & 2.584631 & 3.999764 & 8.318145 \\
\hline 5 & 6 & 0.7075169 & 1.066605 & 1.668016 & 2.942446 & 4.50238 & 9.176312 \\
\hline 5 & 7 & 0.8076738 & 1.211170 & 1.883969 & 3.282990 & 4.993118 & 10.06218 \\
\hline 5 & 8 & 0.89244 & 1.334572 & 2.062933 & 3.568706 & 5.40753 & 10.82776 \\
\hline 5 & 9 & 0.974915 & 1.449774 & 2.230274 & 3.847315 & 5.804434 & 11.49615 \\
\hline 6 & 6 & 0.8362517 & 1.250325 & 1.934166 & 3.357292 & 5.101803 & 10.24216 \\
\hline 6 & 7 & 0.9532409 & 1.417497 & 2.175916 & 3.744367 & 5.625393 & 11.18685 \\
\hline 6 & 8 & 1.053948 & 1.559225 & 2.37994 & 4.067588 & 6.09969 & 11.93585 \\
\hline 6 & 9 & 1.151888 & 1.695543 & 2.583201 & 4.397424 & 6.553556 & 12.80644 \\
\hline 7 & 7 & 1.083447 & 1.595423 & 2.431431 & 4.140509 & 6.192786 & 12.20569 \\
\hline 7 & 8 & 1.198694 & 1.763451 & 2.676427 & 4.537504 & 6.72858 & 13.04854 \\
\hline 7 & 9 & 1.308068 & 1.918944 & 2.897775 & 4.865201 & 7.179527 & 13.83574 \\
\hline 8 & 8 & 1.331007 & 1.948211 & 2.941492 & 4.955321 & 7.298417 & 14.03725 \\
\hline 8 & 9 & 1.450212 & 2.113748 & 3.176776 & 5.310468 & 7.810788 & 14.88969 \\
\hline 9 & 9 & 1.588348 & 2.305376 & 3.451306 & 5.742988 & 8.420277 & 15.94564 \\
\hline
\end{tabular}

Evidently, this involves computation of the generalized hypergeometric function and routines for this are widely available. We used the function hypergeom $(\cdot)$ in the algebraic manipulation package Maple. Tables 4.1 and 4.2 provide the numerical values of $z_{p}$ for $b=1, \beta=1, m=2,3, \ldots, 9$, and $n=m, m+1, \ldots, 9$. 
Table 4.2. Percentage points $z_{p}$ of $Z=|X / Y|$.

\begin{tabular}{|c|c|c|c|c|c|c|c|}
\hline$m$ & $n$ & $p=0.6$ & $p=0.7$ & $p=0.8$ & $p=0.9$ & $p=0.95$ & $p=0.99$ \\
\hline 2 & 2 & 2.002938 & 4.334821 & 11.41224 & 52.30151 & 220.7189 & 5685.296 \\
\hline 2 & 3 & 0.7445086 & 1.382358 & 2.855702 & 8.214106 & 21.04093 & 152.4738 \\
\hline 2 & 4 & 0.4889538 & 0.876642 & 1.710587 & 4.384554 & 10.02126 & 57.84656 \\
\hline 2 & 5 & 0.3832577 & 0.6767803 & 1.286959 & 3.169977 & 6.946125 & 37.36734 \\
\hline 2 & 6 & 0.3251244 & 0.5683113 & 1.068319 & 2.560906 & 5.525674 & 29.00740 \\
\hline 2 & 7 & 0.2832587 & 0.4941505 & 0.9234088 & 2.196231 & 4.668944 & 24.53751 \\
\hline 2 & 8 & 0.2562869 & 0.4435011 & 0.8235854 & 1.936850 & 4.135955 & 21.08758 \\
\hline 2 & 9 & 0.2353905 & 0.4071502 & 0.7504027 & 1.757447 & 3.712460 & 19.04998 \\
\hline 3 & 3 & 1.647264 & 2.842614 & 5.538683 & 15.08887 & 37.62435 & 261.1618 \\
\hline 3 & 4 & 1.085868 & 1.791815 & 3.255642 & 7.909027 & 17.61894 & 98.1088 \\
\hline 3 & 5 & 0.840817 & 1.359837 & 2.407853 & 5.574137 & 11.89116 & 62.47279 \\
\hline 3 & 6 & 0.7106484 & 1.133092 & 1.975254 & 4.480779 & 9.4219 & 49.00014 \\
\hline 3 & 7 & 0.6216468 & 0.988768 & 1.716620 & 3.826405 & 7.905925 & 40.11744 \\
\hline 3 & 8 & 0.558414 & 0.8840209 & 1.517433 & 3.359672 & 6.96206 & 35.21301 \\
\hline 3 & 9 & 0.5107641 & 0.8041508 & 1.376936 & 3.048617 & 6.29827 & 31.90878 \\
\hline 4 & 4 & 1.547561 & 2.487375 & 4.429231 & 10.55419 & 23.34162 & 127.9502 \\
\hline 4 & 5 & 1.207760 & 1.900782 & 3.296398 & 7.461444 & 15.79163 & 83.15728 \\
\hline 4 & 6 & 1.011248 & 1.572540 & 2.678426 & 5.945279 & 12.30920 & 62.89049 \\
\hline 4 & 7 & 0.8819533 & 1.364001 & 2.300262 & 5.037816 & 10.45939 & 53.42483 \\
\hline 4 & 8 & 0.7944737 & 1.222424 & 2.058176 & 4.470943 & 9.197412 & 47.1173 \\
\hline 4 & 9 & 0.7268028 & 1.114706 & 1.866525 & 4.036168 & 8.295475 & 41.95984 \\
\hline 5 & 5 & 1.499379 & 2.331841 & 4.011059 & 9.034731 & 19.02830 & 98.64185 \\
\hline 5 & 6 & 1.257534 & 1.935343 & 3.264778 & 7.174943 & 14.79680 & 76.47803 \\
\hline 5 & 7 & 1.099045 & 1.675479 & 2.801810 & 6.083041 & 12.60114 & 64.14368 \\
\hline 5 & 8 & 0.990024 & 1.500218 & 2.49008 & 5.374516 & 11.01383 & 55.72903 \\
\hline 5 & 9 & 0.906855 & 1.371630 & 2.272028 & 4.863465 & 9.93863 & 50.23113 \\
\hline 6 & 6 & 1.478265 & 2.256043 & 3.782804 & 8.285063 & 17.11076 & 86.97477 \\
\hline 6 & 7 & 1.28456 & 1.946632 & 3.230527 & 6.954553 & 14.26699 & 71.09335 \\
\hline 6 & 8 & 1.155091 & 1.741973 & 2.867585 & 6.141117 & 12.57803 & 62.58595 \\
\hline 6 & 9 & 1.056264 & 1.581389 & 2.598716 & 5.538108 & 11.25649 & 56.78169 \\
\hline 7 & 7 & 1.459679 & 2.198993 & 3.639314 & 7.815963 & 15.95768 & 80.75053 \\
\hline 7 & 8 & 1.30774 & 1.956784 & 3.220993 & 6.878688 & 14.07413 & 71.43641 \\
\hline 7 & 9 & 1.194723 & 1.785849 & 2.932324 & 6.231386 & 12.70544 & 63.87592 \\
\hline 8 & 8 & 1.441835 & 2.152747 & 3.525486 & 7.523065 & 15.35245 & 76.5615 \\
\hline 8 & 9 & 1.320652 & 1.960763 & 3.198957 & 6.790555 & 13.74266 & 69.96122 \\
\hline 9 & 9 & 1.433036 & 2.127872 & 3.470807 & 7.34273 & 14.87503 & 74.51613 \\
\hline
\end{tabular}

We hope these numbers will be of use to the practitioners mentioned in Section 1. Similar tabulations could be easily derived for other values of $p, m, n, b$, and $\beta$ by using the hypergeom $(\cdot)$ function in Maple. 


\section{On the product and ratio of Bessel random variables}

Besides being of practical interest, the above tables can be used to check the accuracy of the results derived in Sections 2 and 3. We estimated the relevant percentage points by simulating samples of size $10^{8}$ from the two Bessel function distributions. The estimates were consistent with the tabulated values up to the third decimal place.

\section{Acknowledgment}

The authors would like to thank the referee and the Associate Editor for carefully reading the paper and for their help in improving the paper.

\section{References}

[1] M. S. Abu-Salih, Distributions of the product and the quotient of power-function random variables, Arab J. Math. 4 (1983), no. 1-2, 77-90.

[2] A. P. Basu and R. H. Lochner, On the distribution of the ratio of two random variables having generalized life distributions, Technometrics 13 (1971), 281-287.

[3] R. P. Bhargava and C. G. Khatri, The distribution of product of independent beta random variables with application to multivariate analysis, Ann. Inst. Statist. Math. 33 (1981), no. 2, 287-296.

[4] M. S. Feldstein, The error of forecast in econometric models when the forecast-period exogenous variables are stochastic, Econometrica 39 (1971), 55-60.

[5] I. S. Gradshteyn and I. M. Ryzhik, Table of Integrals, Series, and Products, 6th ed., Academic Press, California, 2000.

[6] H. G. Grubel, Internationally diversified portfolios: welfare gains capital flows, American Economic Review 58 (1968), 1299-1314.

[7] H. L. Harter, On the distribution of Wald's classification statistic, Ann. Math. Statistics 22 (1951), $58-67$.

[8] D. L. Hawkins and C.-P. Han, Bivariate distributions of some ratios of independent noncentral chi-square random variables, Comm. Statist. A-Theory Methods 15 (1986), no. 1, 261277.

[9] P. J. Korhonen and S. C. Narula, The probability distribution of the ratio of the absolute values of two normal variables, J. Statist. Comput. Simulation 33 (1989), no. 3, 173-182.

[10] S. Kotz, T. J. Kozubowski, and K. Podgórski, The Laplace Distribution and Generalizations. A Revisit with Applications to Communications, Economics, Engineering, and Finance, Birkhäuser Boston, Massachusetts, 2001.

[11] H. J. Malik and R. Trudel, Probability density function of the product and quotient of two correlated exponential random variables, Canad. Math. Bull. 29 (1986), no. 4, 413-418.

[12] G. Marsaglia, Ratios of normal variables and ratios of sums of uniform variables, J. Amer. Statist. Assoc. 60 (1965), 193-204.

[13] T. Pham-Gia, Distributions of the ratios of independent beta variables and applications, Comm. Statist. Theory Methods 29 (2000), no. 12, 2693-2715.

[14] H. Podolski, The distribution of a product of $n$ independent random variables with generalized gamma distribution, Demonstratio Math. 4 (1972), 119-123.

[15] S. J. Press, The t-ratio distribution, J. Amer. Statist. Assoc. 64 (1969), 242-252.

[16] S. B. Provost, On the distribution of the ratio of powers of sums of gamma random variables, Pakistan J. Statist. 5 (1989), no. 2, 157-174.

[17] A. P. Prudnikov, Y. A. Brychkov, and O. I. Marichev, Integrals and Series. Vol. 1: Elementary Functions, Gordon \& Breach Science, New York, 1986.

[18] Integrals and Series. Vol. 2: Special Functions, Gordon \& Breach Science, New York, 1986. 
[19] Integrals and Series. Vol. 3: More Special Functions, Gordon \& Breach Science, New York, 1990.

[20] P. N. Rathie and H. G. Rohrer, The exact distribution of products of independent random variables, Metron 45 (1987), no. 3-4, 235-245.

[21] A. M. Rugman, International Diversification and the Multinational Enterprise, Lexington, Massachusetts, 1979.

[22] H. Sakamoto, On the distributions of the product and the quotient of the independent and uniformly distributed random variables, Tôhoku Math. J. 49 (1943), 243-260.

[23] S. M. Shcolnick, On the ratio of independent stable random variables, Stability Problems for Stochastic Models (Uzhgorod, 1984), Lecture Notes in Math., vol. 1155, Springer, Berlin, 1985, pp. 349-354.

[24] M. D. Springer and W. E. Thompson, The distribution of products of beta, gamma and Gaussian random variables, SIAM J. Appl. Math. 18 (1970), 721-737.

[25] B. M. Steece, On the exact distribution for the product of two independent beta-distributed random variables, Metron 34 (1976), no. 1-2, 187-190 (1978).

[26] A. Stuart, Gamma-distributed products of independent random variables, Biometrika 49 (1962), $564-565$.

[27] J. Tang and A. K. Gupta, On the distribution of the product of independent beta random variables, Statist. Probab. Lett. 2 (1984), no. 3, 165-168.

[28] C. M. Wallgren, The distribution of the product of two correlated t variates, J. Amer. Statist. Assoc. 75 (1980), no. 372, 996-1000.

Saralees Nadarajah: Department of Statistics, College of Arts and Sciences, University of Nebraska, Lincoln, NE 68583-0712, USA

E-mail address: snadaraj@unlserve.unl.edu

Arjun K. Gupta: Department of Mathematics and Statistics, College of Arts and Sciences, Bowling Green State University, Bowling Green, OH 43403-0221, USA

E-mail address: gupta@bgnet.bgsu.edu 


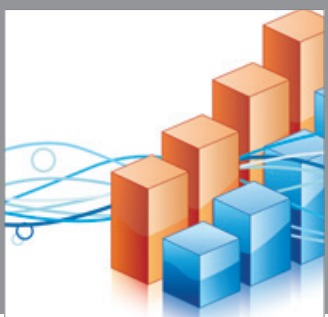

Advances in

Operations Research

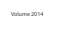

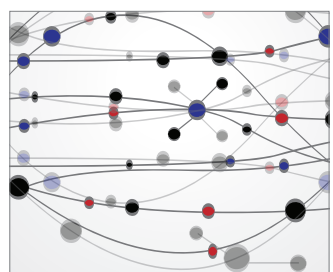

\section{The Scientific} World Journal
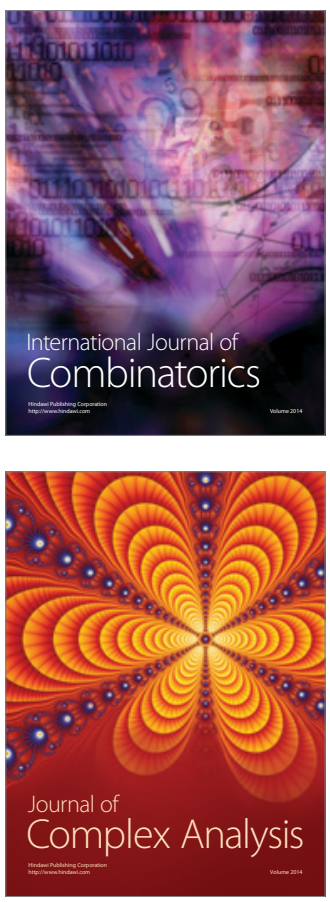

International Journal of

Mathematics and

Mathematical

Sciences
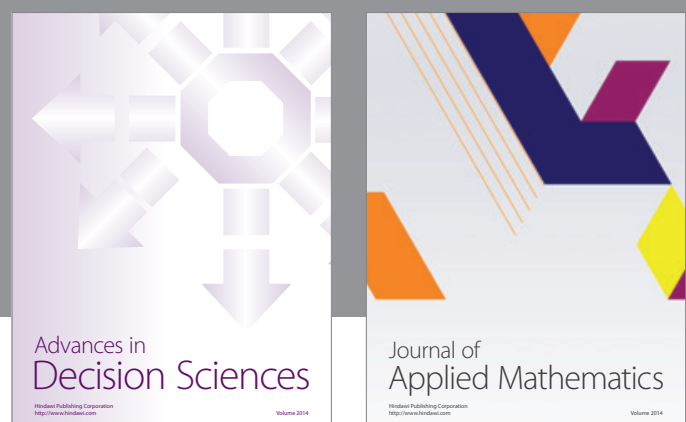

Journal of

Applied Mathematics
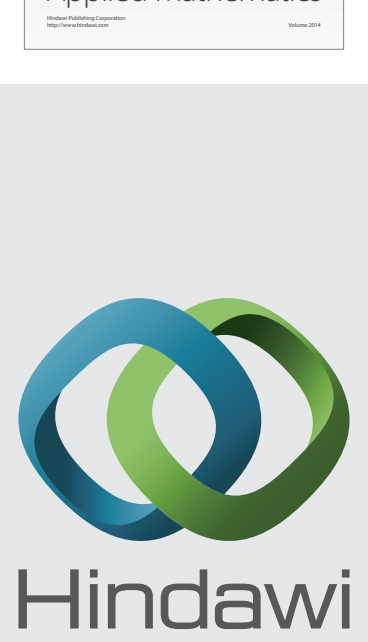

Submit your manuscripts at http://www.hindawi.com
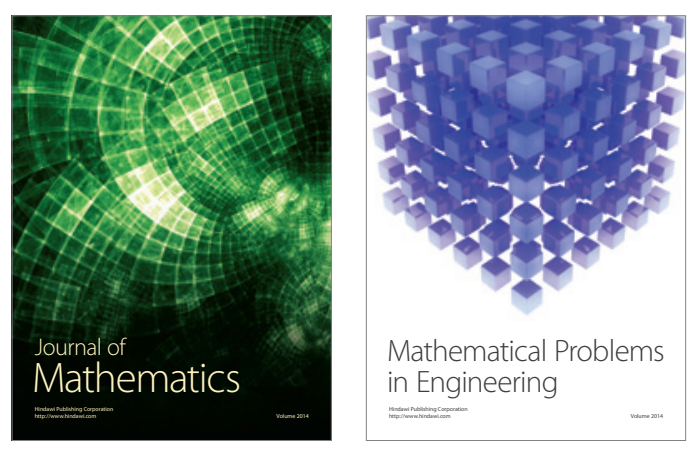

Mathematical Problems in Engineering
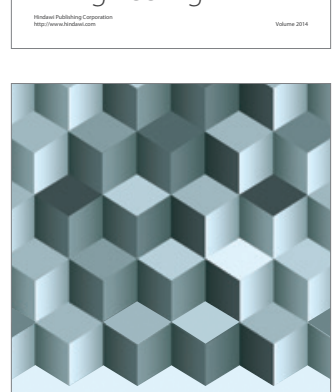

Journal of

Function Spaces
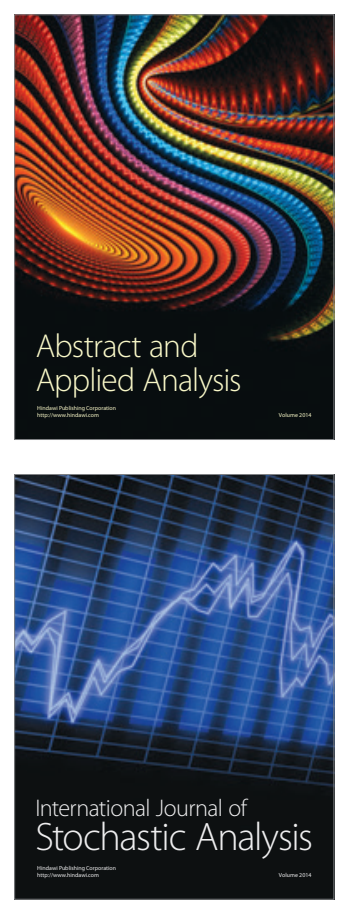

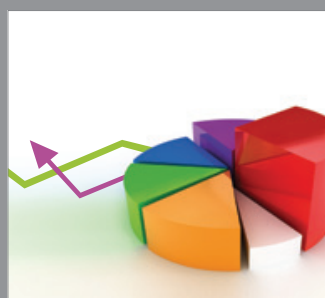

ournal of

Probability and Statistics

Promensencen
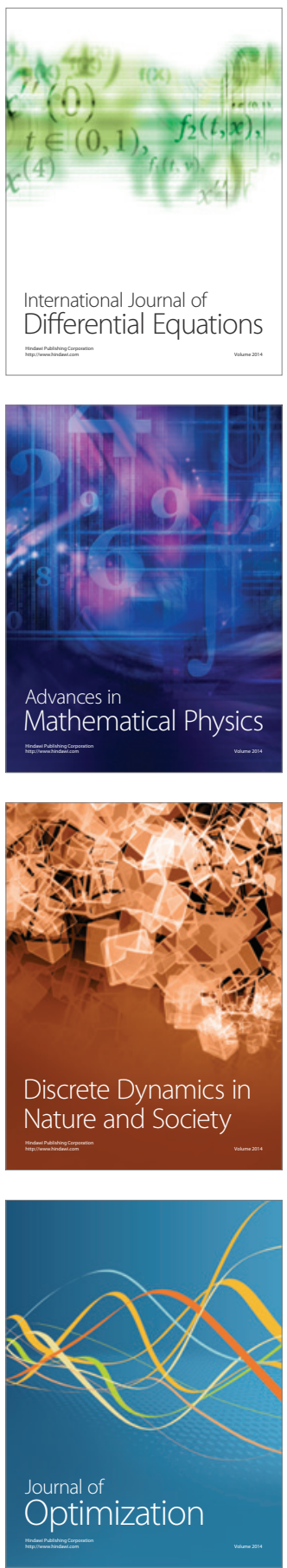